\title{
Integration of nutrition in the antiretroviral therapy scale up plan for Malawi
}

\section{Thomas Bisika ${ }^{1}$, George Mandere $^{2}$}

1. Health Policy and Management School of Health Systems and Public Health, University of Pretoria, South Africa

2. Head of Department, Demographic Unit, Chancellor College, University of Malawi

Corresponding author: Dr. Thomas Bisika, Health Policy and Management School of Health Systems and Public Health, University of Pretoria Box 667, Pretoria 0001, Republic of South Africa. Email: thomas.bisika@up. ac.za

\section{Abstract}

Malawi is one of the countries experiencing an unprecedented $\mathrm{HIV} / \mathrm{AIDS}$ epidemic in an environment where malnutrition is rampant. In 2001 Malawi started providing ARVs to HIV patients. This ARV programme is now being scaled up to cover the whole country. Since underlying malnutrition is associated with adverse ART outcomes, the Malawi government commissioned a study to design a programme for integrating nutrition in the ARV scale up plan. This study used a Participatory qualitative research methodology. The study recommends that nutritional support should include therapeutic feeding, food supplements and take home ration and proposes a clear enrolment and discharge criteria.

\section{Introduction}

\section{HIV/AIDS in Malawi}

Africa is home to about 25 million people living with HIV ${ }^{1}$. Malawi is one of the countries worst hit by the HIV/AIDS epidemic with an infection rate among the childbearing age group of $16.4 \% \%^{2}$. Youth aged $15-24$ claim $46 \%$ of new HIV infections of which $60 \%$ occur among girls. HIV in Malawi is mainly spread through heterosexual $\operatorname{sex}^{3}$ and prevalence among antenatal women ranges from $16 \%$ to $30 \%$. It is estimated that $26 \%$ of the people in urban areas and $11 \%$ in the rural areas live with HIV ${ }^{4}$ although the prevalence is even higher in some rural districts ${ }^{5}$. This suggests that there is a gender, demographic and geographical differential in the HIV/AIDS prevalence within the country. Both infant and under five mortality rates have increased and opportunistic infections like tuberculosis and meningitis are also on the increase in Malawi due to HIV/AIDS ${ }^{6}$.

In order to address the HIV/AIDS problem, the Malawi government adopted an approach aimed at reducing the risk of transmission through intensive mass education on modes of HIV transmission and ways to reduce risk, blood screening, widespread and vigorous use of barrier methods, antibody testing, beneficial disclosure or notification of partners, prevention of mother-to-child transmission (PMTC'T) services, and medical treatment and management of infected individuals including the introduction of ART.

\section{Malnutrition in Malawi}

The prevalence of chronic malnutrition or stunting (measured by height-for-age) among the under 5 children in Malawi is estimated at $49 \%$ and almost half of these are severely stunted. Wasting, measured by weight-for-height index, is estimated at about $6 \%$ among the under five children. Severe wasting is estimated at $1 \%$ and is most common in the 6-23 months age group. The weight-for-age index, a composite index for acute under nutrition (wasting) and chronic under nutrition (stunting), is estimated at about 25\%. Furthermore, about $9 \%$ of women in Malawi have chronic energy deficiency (These are women who had a body mass index (BMI) below $18.5 \mathrm{~kg} /$ square metre $)^{7}$. There has been very little change in the levels of child malnutrition in Malawi since $1992^{8}$.

\section{The link between malnutrition and HIV/AIDS}

The WHO Technical Advisory Group (TAG) on nutrition requirements for people living with $\mathrm{HIV} / \mathrm{AIDS}^{9}$ recommends that in asymptomatic HIV infected adults energy requirements should be increased by $10 \%$ over accepted levels for otherwise healthy persons in order to maintain body weight and a $20 \%$ to $30 \%$ increase for adults during periods of symptomatic or opportunistic infection to maintain body weight.

According to WHO TAG, HIV infected children who are experiencing weight loss require an increased energy of $50 \%$ to $100 \%$ over established requirements for otherwise healthy uninfected children in order to achieve catch-up growth.

\section{Nutrition and antiretroviral therapy}

Wasting and weight loss remain common problems among $\mathrm{HIV}$-infected persons. Already weight loss has been documented as an independent predictor of mortality and lower CD4+ cell counts ${ }^{10}$. Research has shown that low serum micronutrients levels are common before the introduction of highly active antiretroviral therapy $(\text { HAART })^{11}$, and these deficiencies have been associated with adverse HAART outcomes . Other studies have also concluded that malnutrition at the time of starting ART is significantly associated with increased mortality ${ }^{12}$. Against this background WHO TAG recommends that nutritional intervention should be an integral part of all HIV treatment programmes citing that improved attention to diet and nutrition may enhance ART acceptability, adherence and effectiveness.

\section{ART scale up in Malawi}

As one strategy to support people living with HIV and AIDS (PLWHA), Malawi started providing antiretroviral (ARV) therapy at selected hospitals in 2001. Patients originally accessed the ARV drugs by paying fees. Due to poverty, many eligible patients could not afford to pay for the drugs, as a result, the ART service benefited fewer patients which was in violation of the principle of equity in health care.

To address this problem the government instituted a policy which reduced and/or completely removed payment for ARVs. In line with this policy, the Malawi government through the Ministry of Health and in collaboration with the National AIDS Commission (NAC) is scaling up ART service to many public, mission, non-governmental organization (NGO) and private hospitals. As part of expansion of the ART service, the Ministry of Health has been training health workers who will be involved in the provision of the ART services. As a result of the ART service scaling up, it is estimated 
that more than 100,000 people in Malawi are now on highly active antiretroviral therapy (HAART). This achievement has contributed towards the WHO goal of getting 3 million people in developing countries on ARV therapy by the end of 2005 which was code named the ' 3 by 5 ' Initiative.

The purpose of this study was to identify a nutrition intervention that would complement the ARV scale up and draw a consensus on enrolment and discharge criteria.

\section{Method}

The study was descriptive in nature and used both qualitative and quantitative approaches. The study was conducted in 2005 and 2 health facilities in the northern region; 3 in the central region; and 3 in the southern region were selected. The health facilities were a combination of both government and mission hospitals. Initial key informant interviews, meetings and workshops with HIV/AIDS and nutrition focal persons representing WFP, WHO, UNICEF, FAO and Ministry of Health as well as service providers was conducted to discuss and reach a common understanding on the approach, issues to be addressed, health institutions to be visited, groups of people to be interviewed and schedule of activities. The meetings collected and gathered documents related to HIV/ AIDS and nutrition to ensure that the proposed activities were pertinent and appropriately focused.

\section{Data collection}

Participatory qualitative research methodologies was employed for data collection. Primary data was collected during fieldwork through stakeholders' consultative workshops, in-depth interviews with health personnel and representatives of community based organization and NGOs as well as focus group discussions with members of beneficiary communities. The approach was preferred to ensure that methods were triangulated so that when there is consensus the results could be accepted with much confidence. The sero-status of the people who participated in the focus group discussions was unknown. Individual interviews with a convenient sample of HIV patients on ART, some of whom were beneficiaries of nutrition programmes, was also conducted. These HIV patients were identified through referral by the service providers and community based organizations. No anthropometric measurements were taken for the ART beneficiaries which is a limitation in that the nutrition status of the beneficiaries could not be established. Only health facilities that had introduced ART were visited by design to ensure relevance of the questions that were being asked. Both public and private facilities from the three regions were visited to ensure adequate representation.

\section{Data management, processing and analysis}

During fieldwork, great care was exercised to ensure data reliability and validity. In this regard a debriefing session was organized at the end of FGDs to share salient issues that came up in the separate group discussions. The wrap up meetings offered an opportunity to the research team to validate the data collected. Transcripts of the FGDs were prepared using field notes and audio-cassettes. Individual ART beneficiary interviews were also transcribed. For ethical reasons, all these transcriptions were kept confidential at all times and the interviews were conducted in full privacy after
Table 1 Characteristics of ART beneficiaries

\begin{tabular}{ll} 
Background Characteristics & Total \\
\hline Sex & \\
\hline Male & 24 \\
Female & 32 \\
\hline Age Group & \\
\hline $15-19$ & 2 \\
$20-24$ & 4 \\
$25-29$ & 5 \\
$30-34$ & 12 \\
$35-39$ & 10 \\
$40-44$ & 10 \\
$45-49$ & 5 \\
$50+$ & 8 \\
\hline Marital Status & \\
\hline Never married & 9 \\
Married & 27 \\
Widowed & 16 \\
Divorced & 4 \\
\hline Education & \\
\hline Primary & 31 \\
Secondary & 21 \\
Tertiary & 4 \\
\hline Employment & 24 \\
\hline Employed & 32 \\
\hline Not employed &
\end{tabular}

obtaining an informed verbal consent. An approval letter was obtained from the Ministry of Health, which is the secretariat of the Malawi National Health Sciences Research Committee, before commencement of the fieldwork.

Content analysis of the qualitative information was done using the manual search and code method while percentages were calculated using a simple calculator. Computation of statistics like p-value, standard deviation and confidence intervals was not done.

\section{Results}

A total of 56 patients on ARV treatment participated in the individual interviews and 18 FGDs were conducted. These were basically meant to appraise the nutrition intervention as proposed by the health officials. Some respondents came from within the surrounding areas of the health facilities visited while others came from distant places. Table 1 presents the background characteristics of the ART beneficiaries who took part in the study. According to this table, thirty-two respondents were females while 24 respondents were males. Most of the ART beneficiaries interviewed (32) were aged between 30 and 44 years and the majority were married. A substantial number (16) had lost their spouses. Thirty- one (31) respondents had primary education, 21 had secondary education while only 4 had reached tertiary level. Thirtytwo respondents reported that they were not employed. Among this group, some reported they had stopped formal employment because they were physically unfit to continue working and were dependent, others were engaged in small- 
scale businesses as a coping strategy.

Almost a third of the ART patients who participated in the study reported receiving nutritional support although most of them were of the opinion that this support was inadequate. About $85 \%$ of the ART beneficiaries reported that they could not afford a balanced diet due to illness and $17 \%$ of them reported that they were times when they had to miss treatment due to lack of food. Most of them reported that they needed nutrition support and almost all of them (90\%) mentioned that this support was required during the time they were sick and acknowledged that once they were fine they could find food on their own without problems. $15 \%$ of ART beneficiaries reported that they can find their own food but that they would still need advice on how to eat well at various stages of the infection.

The ART beneficiaries mentioned maize, sugar, cooking oil, rice, fish, meat, beans, likuni phala, milk, plumpy nut, ground nuts, fruits and vegetables as some of the food items they were in need of. In terms of distribution method, most of the ART patients $(70 \%)$ would prefer receiving food items at the clinic, 10 percent in the community and about one fifth would not mind where they collected their supplies from.

\section{Institutional capacity to implement nutrition support intervention}

All health facilities visited had initiated and were providing ART services. This was expected because only facilities that had initiated ART were included in the study. The number of patients on ARV therapy ranged from 135 at Mulanje Mission Hospital to 1500 at Lighthouse at Kamuzu Central Hospital. The ART providers reported that their facilities have unmet demand for ART services. At some ART clinics, the clinicians reported that new patients in WHO stage 3 or 4 of the HIV infection are put on waiting list due to inadequacy of ART supply and that some patients eligible for ARV treatment do not present themselves to the ART clinics because of long distance to the hospital and fear of stigma and discrimination.

In addition to providing ARV drugs, the health facilities were reported to be providing VCT services, treatment of opportunistic infections, nutrition education and health education. Only a few health facilities reported to be providing PMTCT services. Most of the health facility based staff reported that they provide monitoring and follow-up services of patients at the hospitals and in communities.

Out of the eight health facilities visited, Ekwendeni Mission Hospital, Chiradzulu District Hospital, Mulanje Mission Hospital and African Bible College Clinic reported that they provide nutrition support to HIV / AIDS patients. Food items provided to patients included soya, maize, BP5 biscuits, likuni phala, cooking oil and beans. Sources of the food support that were cited by the hospital staff were Christian Health Association of Malawi (CHAM), WFP and individual wellwishers. The amount of food items that patients received varied from one health facility to another and within the health facility it varied from time to time depending on availability of supplies. Distribution of food rations was mostly done at the health facilities although some health facilities reported that they also distributed the food in communities with assistance from HBC volunteers. At the health facility food was distributed either by health providers or NGOs. For example, at Chiradzulu District Hospital food distribution was done by MSF which is an international NGO at the facility and the food was supplied by WFP. In some cases, it was reported that food assistance was sporadic because of shortage of supplies. Cases of severely malnourished (BMI less than 16) ART patients being referred to the maternal and child health $(\mathrm{MCH})$ programme to access food meant for pregnant and lactating mothers were reported. According to the service providers a standard food ration is provided to patients irrespective of the stage of the HIV infection.

All health facilities visited expressed willingness to be involved in providing food rations to patients if food was given to them. The health providers reported that patients have complained about lack of food and failure to take daily pills due to unavailability of food. The majority of the health facilities reported that, at present, they have limited capacity or do not have the capacity to implement the nutrition support intervention. Food storage facilities were not available and in health facilities where they were available, they were small. Health facilities were also limited in terms of human resources. Due to shortage of manpower, clinicians and nurses interviewed said they were already overstretched and their involvement in the nutrition support intervention would be an additional workload. Limited capacity was also expressed in the area of transport to conduct followup and monitoring visits to patients in their respective communities.

In the focus group discussions, community volunteers indicated that they would participate in the nutrition support intervention in areas of food distribution, monitoring food availability and utilization of food among patients and affected households. They could also help in conducting educational campaigns and referrals of patients to hospitals. In some communities visited, the volunteers were already involved in similar activities with other programmes. For example, it was reported that the volunteers were involved in food distribution to vulnerable people such as the elderly, orphans and chronically ill patients. Their experience was that if there were no clear enrolment criteria and/or interference from community leaders, some food items would end up being given to wrong people. They, however, cautioned that they forego their own work in order to do voluntary work an aspect that they said affected their livelihood which raises the issue of sustainability. If they were to implement the nutrition support intervention effectively, the volunteers expressed need for capacity building in areas of patient identification, transport (bicycles) and nutrition counselling among others.

\section{Nutrition support to ART beneficiaries, admission and discharge criteria}

The key informants that were interviewed proposed three types of nutrition support: therapeutic and supplementary feeding to the ART beneficiaries and food ration to the affected households. There were also suggestions for enrolment and discharge criteria for the proposed nutrition support programme. These were consistent with the views of the beneficiary communities who were of the view that lack of such criteria have undermined the effectiveness of similar programmes in the past. Therapeutic feeding and supplementary feeding according to health officials and providers should be provided to patients who are experiencing 
weight loss as a result of malnutrition.

The key informants suggested that therapeutic feeding should be provided to patients based on the following criteria:

(i) Adults: BMI less than 16.0 (BMI $<16$ refers to severe malnutrition ${ }^{13}$ while BMI $<17 \mathrm{~kg} /$ square metre constitutes moderate malnutrition ${ }^{12}$

(ii) Pregnant and lactating mothers with mid upper arm circumference (MUAC) less than $20 \mathrm{~cm}$

(iii) Children: weight-for-height less than 70\%, MUAC less than $11 \mathrm{~cm}$

According to the key informants, patients who meet the above criteria should be given F75, F100 or other Ready to Use Therapeutic Food (RUTF) such as plumpy nut. According to the health workers, F75 should be provided to stabilize the patient during hospital stay and this is normally given for 1 week. Thereafter, the patient should be given F100, RUTF or plumpy nut until the patient is stabilized.

The discharge criterion for the therapeutic feeding intervention was suggested as follows:

(i) Adults: $\mathrm{BMI}>18.5$

(ii) Pregnant and lactating mothers: MUAC of between 20-22cm,

(iii) Children: weight for height between $70-80 \%$ and MUAC $11-12 \mathrm{~cm}$

According to the health providers, beneficiaries usually meet the discharge criteria after approximately four weeks. These people directly qualify for the supplementary feeding programme.

For the supplementary feeding the key informants suggested that this should be given to patients based on the following enrolment criteria:

(i) Adults: BMI between 16.0 and 18.5

(ii) Pregnant and lactating mothers: MUAC less than $22 \mathrm{~cm}$

(iii) Children: weight-for-height less than 80\%, MUAC less than $12 \mathrm{~cm}$

According to the key informant, the adult patients in above conditions should be given $4.5 \mathrm{kgs}$ of fortified Likuni Phala with $10 \%$ sugar, $500 \mathrm{ml}$ vegetable oil issued as premix every two weeks or soya flour (ie $9 \mathrm{~kg}$ per month mixed with 1 litre of vegetable oil) or 1 bottle of plumpy nut $260 \mathrm{~g}$ per day as has always been the practice in facilities where nutritional support is on-going. These amounts must be adjusted for children. The key informants reported that presently this nutrition support is provided through health centres but it is possible to provide the supplementary feeding commodities through community home based care and home based organizations. Some health facilities reported that they provide supplementary feeding for a maximum of 4 months unless discharge criteria are reached before that.

The discharge criteria for supplementary feeding ware suggested by the key informants as follows:

(i) Adults: BMI $>18.5$

(ii) Pregnant and lactating mothers: MUAC $>22.5 \mathrm{~cm}$

(iii) Children weight for height $>85 \%$, MUAC $>12.5 \mathrm{~cm}$
Food Rations were recommended for those ART beneficiaries who have been discharged from the supplementary feeding and are food insecure. The respondents proposed that a take home food ration should be provided for both the patient and affected household. The respondents feared that if food ration was only provided for the patient, the food supplement might end up being shared with other household members. To enrol affected households with ART beneficiaries in food assistance programme, the following criteria were proposed:

(i) Patient is unemployed

(ii) Patient is physically weak/bedridden

(iii) No member of household is capable of providing food for the household

(iv) Widow

(v) Orphan

(vi) Older persons

(vii) Household/patient has no stable source of income

(viii) Poor crop harvest especially maize

(ix) Low income though employed

The discharge criteria for the food assistance to the affected households should be the following:

(i) Resumption of employment by ART beneficiary

(ii) Death of the ART beneficiary

(iii) Household/ patient is food secure

(iv) One or more members of the household are providing food for the household

(v) Change of place of residence by patient

(vi) Household enrolled in another food assistance programme

According to the informants one standard ration comprising of $50 \mathrm{~kg}$ of maize, 4 litres of cooking oil and $10 \mathrm{~kg}$ of pulses per months and it is estimated that this will be provided for six months. This ration is considered standard because it does not take into account the household size.

\section{Cost analysis of the nutrition support}

It was estimated that 44,000 households would require food ration over a period of six months in one year. This means that the total household food ration required was 13,200 metric tons of maize which would cost MK224.4 million, 2,640 metric tons of pulses at an estimated cost of MK316.8 million and 1.32 million litres of cooking oil that would normally cost MK356.4 million.

The type of nutrition support for therapeutic and supplementary feeding would vary from one beneficiary to another depending on the stage of HIV progression. It was generally assumed that the cost of the nutritional support to ARV patients would be 20 percent of the household rations (MK179.5 million over a period of six months).

\section{Current nutrition support (Food Basket and Ration)}

The ART clinicians reported that food assistance to HIV/ AIDS patients including those on ARV treatment varied in amounts depending on food availability. At a central level debriefing meeting, stakeholders indicated that one standard food ration might not be appropriate to address the nutritional 
unable to digest certain foods that they eat all the time. The

\begin{tabular}{|c|c|}
\hline Symptom & Suggested Strategy \\
\hline \multirow[t]{2}{*}{ Severe diarrhea } & $\begin{array}{l}\text { Drink liquids frequently (oral re-hydration } \\
\text { solution, diluted fruit juices) }\end{array}$ \\
\hline & $\begin{array}{l}\text { - Eat bananas, mashed fruits, soft rice and } \\
\text { porridge }\end{array}$ \\
\hline \multirow[t]{5}{*}{$\begin{array}{l}\text { Nausea and } \\
\text { vomiting }\end{array}$} & $\begin{array}{l}\text { Eat simple boiled foods, potatoes, beans, } \\
\text { porridge }\end{array}$ \\
\hline & - Drink diluted fruit juices, clear liquids \\
\hline & - Eat soups and crackers (non-sweet biscuits) \\
\hline & $\begin{array}{l}\text { - Flat cola or coke which is made by allowing the } \\
\text { coke to sit exposed to air until the bubbles are } \\
\text { gone }\end{array}$ \\
\hline & - Avoid foods that have strong aroma \\
\hline
\end{tabular}

Sore mouth and - Avoid sugary, fried and spicy foods

throat - Chew raw garlic

- Eat soft foods, porridge, yogurt, soups

- Rinse with warm, salt or baking soda

Fever and loss - Drink high energy, high protein liquids such as of soured milk (chambiko), thobwa, and low sugar appetite fruit juices

- Eat small portion of preferred foods that have pleasing aroma and texture

- Eat nutritious snacks like boiled pasteurized and soured milk, nuts, bean patties, bananas, pineapples, boiled cassava and sweet potatoes

- Drink liquids often (2 litres per day)

Weight loss - Eat foods such as bananas, avocado, nuts, butter, cheese, potatoes, fatty fish, porridge, protein.

Loose bowels - Eat bananas, mashed fruits, soft rice and porridge

- Eat small meals more often

- Decrease high fat foods, take frequent fluids and reduce dairy products to see if they are the cause

- Avoids foods insoluble fibre (roughage)

Fat $\quad$ Eliminate oils, butter, margarine and foods that malabsorption contain/or are prepared with them

- Eat only lean meat, fruits and vegetables with other low fat foods

Fatigue and - Eat fresh foods that don't require lethargy preparation

- Drink high energy, high protein liquids

- Set aside time each day for eating

- Have someone precook foods to save time and energy in preparation (care with reheating)

Constipation - Eat foods in high fibre like mgaiwa, fruits with edible skin, whole-wheat bread, green leafy vegetables, prunes

- Drink plenty of fluids (at least 2 litres clean water per day)

- Do not use enemas unless directed by doctor

- Drink Aloe Vera juice

- Exercise daily such as taking a walk

Source: Ministry of Health 2004

needs of HIV/AIDS patients in different stages of the HIV infection. As a result of HIV/AIDS, some patients are medicines that they take also make them feel sick and have some negative effects on their stomachs. Other patients have infections in their mouths that hurt when swallowing food. Other patients may feel too tired to eat. They proposed that ART beneficiaries should be given advice on appropriate nutrition depending on stage of HIV infection and specific symptoms and opportunistic infections experienced. The suggested nutrition strategy according to $\mathrm{MOH}$ (2004) for patients with various HIV/AIDS symptoms and signs is given in Table $2^{14}$.

\section{Distribution points for the nutrition support}

During the study, ART beneficiaries and non-beneficiaries were asked about the most convenient sites to provide nutrition support. Two delivery points namely health facility and community were proposed.

(a) Clinic based support

The health facility was proposed as an ideal distribution site for the reasons that:

(i) ART beneficiaries go to the ART clinics to collect their drugs. During such visits the patients could be provided with the required food commodities. This would help increase treatment compliance.

(ii) The food commodities would only be given to rightful beneficiaries unlike in the community where there might be bias by authorities responsible for the food distribution;

(iii) ART beneficiary's right to confidentiality would be guaranteed and respected.

(iv) In the communities, food storage and security would be problematic assuming that food is not distributed on the same day it is brought to the community.

(v) In the rainy season, most roads especially in the rural areas become impassable. As a result, food might not be brought to distribution sites in the communities.

The major limitations of clinic-based food support were the capacity of the health facilities (storage facilities and human resources) to handle the nutrition component.

\section{(b) Community based support}

An alternative to the health facility is to designate community distribution centers. The community food distribution would involve HBC volunteers and representative(s) from ART clinic and/or an implementing NGO. A programme could be worked out between the HBC volunteers and health facility/NGO personnel and WFP for the food distribution. To avoid problems of storage facilities and possible food pilferage, respondents suggested that the food should be brought to the community on the day of distribution. With such an arrangement, patients would be able to receive their food rations within walking distances and the communities at large would have a sense of ownership of the programme.

The major problem of food distribution in the community was mentioned to be stigma. As ART beneficiaries collect their food rations, they will be identified by other community members that they are HIV positive and on ARV treatment. For fear of the actual or anticipated stigmatization and discrimination, some eligible beneficiaries will be 
uncomfortable to collect their rations from the community distribution centers. The concern for stigma was raised by majority of ART beneficiaries although some did not mind receiving the food in the communities.

\section{Discussion}

Most of the HIV patients on ART indicate the need for nutritional support during the time they are ill. This is not surprising because unintentional weight loss of more than $10 \%$ is an indication for the initiation of ART which suggests that most of the patients on ART may have already experienced wasting. What was surprising in this study was that most of the patients attributed the need for nutritional support to inability of their households to find food due to illness of a bread winner and not directly to ART. According to the ART beneficiaries, therefore, even those HIV patients who are not on ART need nutritional support. This seems to support the inclusion of unintentional loss of more than $10 \%$ of body weight in the admission criteria for nutritional support.

The HIV patients on ART were not familiar with therapeutic feeding. Most of them knew nutritional support from a household food security perspective. This suggests the need for more nutritional education so that patients should be able to value both therapeutic and other forms of nutritional support. The type of food items suggested by the ART beneficiaries vindicates this point. It must be pointed out at this point that the nutritional interventions proposed by ART beneficiaries and those suggested by the key informants were complimentary.

The HIV patients on ART were generally sceptical about food distribution within the community. This suggests that stigma is still rampant at community level. The other explanation is that the patients collect their ARVs at the clinic and this is also where they are monitored so they may have no difficulties collecting food items from the clinic. The only danger is that some of the food items are bulky and may be difficult to be carried by a sick person. To address this problem it is suggested that a coupon system be used where the patient could be given coupons equivalent to the food required. An arrangement could then be made with existing suppliers like Agricultural Development and Marketing Corporation (ADMARC), Mc Connel \& Company, People Trading Centre (PTC), Shoprite and Metro among several others.

It must be observed at this point that therapeutic and supplementary feeding are similar terms if we consider that both are given to those nutritionally deficient. It is therefore possible that supplementary feeding as suggested by the key informants refers to complementary feeding. Furthermore what is being described as food ration can be considered as supplementary since it is partly going to be used by the patients.

\section{Conclusion}

There is consensus among the different stakeholders about the need for good nutrition for HIV patients on ARV drugs although only a few facilities are already providing nutrition support. The stakeholders, therefore, proposed that a nutritional support programme for patients on ARV drugs should be implemented. The nutritional support recommended included therapeutic and supplementary feeding as well as food ration for the affected households. These could be delivered either at the clinic or in the community although ART beneficiaries are likely to be stigmatized at the community level. The recruitment criteria proposed for supplementary feeding were BMI less than 18.5 for adults, MUAC less than $22 \mathrm{~cm}$ for pregnant and lactating mothers and weight for height less than $80 \%$ or MUAC less than $12 \mathrm{~cm}$ for children. The suggested recruitment criteria for therapeutic feeding were BMI less than 16.0 for adults, pregnant and lactating mothers with Mid-Upper arm circumference (MUAC) less than $20 \mathrm{~cm}$ and Children whose weight-for-height is less $70 \%$ and MUAC less than 11 $\mathrm{cm}$. Attainment of these thresholds was recommended as discharge criteria.

Nutritional support will require increasing the number of facilities that are providing nutritional support and addressing problems such as inadequate storage space, logistics, irregular food supplies and human resources. There are a number of partners who have already expressed their willingness to conduct nutrition education and capacity building which can be used to expand the programme. From a hospital point of view, this type of intervention may entail capacity building in the area of therapeutic feeding. Finally since this programme has been rolled out for over 2 years it is important to commission a mid-term review to assess whether the programme is exerting the desired impact.

\section{Acknowledgment}

This paper is based on the study that was funded by the World Food Programme (WFP).

\section{References}

1. UNAIDS. 2006. 2006 Report on the global AIDS epidemic

2. UNAIDS. Malawi - Epidemiological Fact Sheet on HIV/AIDS and Sexually Transmitted Infections, 2004 Update [cited 8 Oct 2005]. Available from: http://www.unaids.org/html/pub/publications/fact-sheets01/ malawi_en_pdf.htm

3. Barden-Ofallon J, deGraft-Johnson J, Bisika T, Benson A, Tsui A. Factors associated with HIV/AIDS Knowledge and Risk Perception in Rural Malawi. AIDS and Behavior; 8(2):131-140.

4. National AIDS Commission. National HIV/AIDS Policy: A Call for Renewed Action. 2003

5. Hoffman IF, Tsui A, de Graft-Johnson J, Bisika T, Feluzi H, Kazembe PN. Reducing the STI burden in Rural Malawi : STD screening, Case and Contact Treatment, and Condom Promotion. University of North Carolina: Chapel Hill. 2003:1.

6. Malek A, Bisika T. Food Assistance to HIV/AIDS Infected and affected Individuals: A Revised Programme. WFP/UNAIDS: Lilongwe. 2001:6.

7. Macro International. Malawi Demographic and Health Survey. 2000

8. Macro International. Malawi Demographic and Health Survey. 1992

9. WHO (2003), Nutritional requirements for people living with HIV/AIDS: report of a technical consultation 13-15 May 2003

10. Mangili A, Murman DH Zampini AM et al. Nutrition and HIV infection: review of weight loss and wasting in the era of highly active antiretroviral therapy from the nutrition for healthy living cohort. Clinical Infectious Diseases 2006; 42: 836-842.

11. Jones CY, Tang AM, Forrester JE et al. Micronutrient levels and HIV disease status in HIV-infected patients on highly active antiretroviral therapy in the Nutrition for Healthy Living cohort. Journal of Acquired Immune Deficiency Syndrome 2006; 43(4): 475-482.

12. Patton NI, Sangeetha S, Earnest A et al. The impact of malnutrition on survival and the CD4 count response in HIV-infected patients starting antiretroviral therapy. HIV Medicine. 2006; 7: 323-330.

13. Administrative Committee on Coordination/Sub-Committee on Nutrition - (ACC/SCN) The Un System's Forum for Nutrition available at http:// www.unsystem.org/scn/archives/rwns04/ch14.htm [accessed on June 30, 2008]

14. Ministry of Health. 2004. Treatment of AIDS: The two year plan to scale up Antiretroviral Therapy in Malawi 2004-2005 